\title{
Which is the most reliable diagnostic modality for detecting locally residual or recurrent laryngeal squamous cell carcinoma after (chemo)radiotherapy?
}

\author{
Peter Zbären • Remco de Bree • Robert P. Takes • \\ Alessandra Rinaldo • Alfio Ferlito
}

Received: 5 May 2013/ Accepted: 8 May 2013/Published online: 21 May 2013

(c) Springer-Verlag Berlin Heidelberg 2013

Radiotherapy and, more recently, chemoradiotherapy have become important treatment modalities in the treatment of laryngeal squamous cell carcinoma (LSCC). In early stage LSCC, it is an alternative to minimally invasive (microscopic laser) surgery or partial laryngectomy and in advanced stage disease chemoradiotherapy is an organpreserving alternative for laryngectomy [1-4]. Therefore, the head and neck surgeon must frequently deal with recurrent or residual LSCC. The recurrence rate in T2-T4 LSCC after (chemo)radiotherapy is reported between 25 and $50 \%$ [5]. The identification of recurrent or residual disease may be difficult. Clinical symptoms and signs secondary to radiation toxicity such as hoarseness, dysphagia, respiratory distress and pain are quite similar to those of recurrent carcinoma. Moreover, the growth pattern of recurrent LSCCs is different from that of primary carcinomas [6]. Many recurrences, even after initial T1 or T2

This paper was written by members of the International Head and Neck Scientific Group (www.IHNSG.com).

\section{P. Zbären}

Department of Otorhinolaryngology-Head and Neck Surgery, University Hospital, Berne, Switzerland

\section{R. de Bree}

Department of Otolaryngology-Head and Neck Surgery,

VU University Medical Center, Amsterdam, The Netherlands

\section{R. P. Takes}

Department of Otolaryngology-Head and Neck Surgery, Radboud University Nijmegen Medical Center, Nijmegen, The Netherlands

A. Rinaldo $\cdot$ A. Ferlito $(\bowtie)$

ENT Clinic, University of Udine, Piazzale S. Maria della

Misericordia, 33100 Udine, Italy

e-mail: a.ferlito@uniud.it carcinomas, present with multicentric tumor foci, localized below an intact mucosa.

The distinction between recurrent or residual carcinoma and radiotherapeutic sequelae such as fibrosis, edema, soft tissue necrosis, perichondritis and cartilage necrosis can be challenging either by endoscopy with biopsies or by imaging studies.

The focus of this Editorial is on (1) which is the appropriate diagnostic modality to distinguish recurrent or residual disease from post-(chemo)radiotherapy changes and (2) recurrent tumor assessment and cr-T-classification, which is a prerequisite for voice-preserving salvage surgery.

\section{Endoscopy under general anesthesia with biopsy}

The evaluation of the irradiated larynx is probably the most difficult of all head and neck sites. An analysis of Brouwer et al. [7] in 2004 of 207 direct laryngoscopies under general anesthesia in 131 patients with suspected recurrent LSCC showed a recurrence rate of $72 \%$; 70 of the first 131 laryngoscopies performed were negative and in $22(31 \%)$ of them a recurrence was diagnosed in subsequent laryngoscopies within 6 months. In another 11 patients, recurrence was proven more than 6 months after a first negative laryngoscopy - the majority of these within 12 months. A total of 81 laryngoscopies were needed to detect these 33 recurrences. In the group of 37 patients finally classified as disease free, a total of 65 laryngoscopies were performed. This study indicates that a considerable number of futile laryngoscopies to exclude or to detect recurrent disease are performed. In case of suspicion of recurrent carcinoma, the sensitivity and specificity of biopsies are reported to be 25 and $97 \%$, respectively by Viani et al. [8] in 1991 and 51 
and $100 \%$, respectively by Terhaard et al. [9] in 2001. In comparison, the sensitivity and specificity of the surgeon's opinion that recurrent cancer was present, was reported to be 46 and $98 \%$ [10]. Thus, the opinion of an experienced head and neck surgeon is quite equal to that of an endoscopic evaluation with biopsies. Therefore, an imaging study allowing more accurate post-(chemo)radiotherapy evaluation would potentially be cost-effective to select patients who might benefit from direct laryngoscopy under general anesthesia with biopsies.

\section{Conventional magnetic resonance imaging (MRI) and computed tomography (CT)}

Brouwer et al. [5] performed a "systematic review on the accuracy of imaging tests in the diagnosis of recurrent laryngeal carcinoma after radiotherapy". One of the surprising facts in the search for literature was the observation that there was little literature on the specific question. According to their selection criteria, no eligible studies on CT and MRI imaging were found. The most frequent reason of ineligibility was that studies comprised various tumor locations rather than LSCC only [5]. In two studies, evaluating if pretreatment and post-treatment CT or MRI imaging can predict response in patients treated with radiotherapy, the authors concluded that pre-treatment imaging risk profiles as well as post-radiotherapy imaging evaluation can identify patients at high risk for developing local failure [11, 12]. According to Hermans [13], baseline and follow-up CT may improve detection of recurrent LSCC after radiotherapy. The baseline CT or MRI imaging should be obtained about 3-6 months after the end of therapy. Nevertheless, the problem of baseline imaging studies is that not only tumor recurrences account for imaging changes during follow-up, but also the progression of tissue changes induced by (chemo)radiotherapy. Most authors concur that neither CT nor conventional MRI imaging can accurately distinguish between recurrent carcinoma and radiotherapeutic sequels in most cases $[7,14,15]$. In a prospective evaluation of 46 patients with suspicion of recurrent laryngeal or hypopharyngeal cancer, the sensitivity, specificity and accuracy in detecting recurrent disease by conventional MRI was 72, 57 and $63 \%$, respectively [16].

\section{Fluorodeoxyglucose positron emission tomography-CT (FDG-PET-CT)}

Most studies evaluating of the ability of FDG-PET-CT in detecting recurrent or residual LSCC after (chemo)radiotherapy include tumors of various anatomical head and neck sites. Furthermore, many studies evaluated the treatment response of (chemo)radiotherapy and were not conducted on patients with suspicion of recurrent or residual LSCC. Most studies were performed with FDGPET without CT implying a lack of anatomic details. Combined FDG-PET/CT seems to be more accurate in detecting recurrent head and neck squamous cell carcinoma (HNSCC) than FDG-PET alone [17, 18]. However, Gupta et al. [19] found in a meta-regression analysis no significant difference between stand-alone PET and integrated PET/ CT. Nevertheless, most studies show a benefit of PET(CT) in detecting recurrent or residual HNSCC. Only a limited number of studies included laryngeal carcinoma patients. A study of 30 patients with suspicion of LSCC recurrence showed a sensitivity, specificity and accuracy of 88,82 and $83 \%$, respectively, in detecting recurrent disease by FDG-PET [5]. Terhaard et al. [9] prospectively analyzed 75 patients with suspicion of recurrent LSCC. The sensitivity and specificity of the first FDG-PET was 92 and $63 \%$, respectively. In all patients with a negative FDG-PET scan, laryngoscopies under general anesthesia performed shortly after the FDG-PET scan were negative and patients remained disease free for at least 1 year. The authors concluded that no biopsy was needed if the FDGPET scan was negative.

In a systematic review, the pooled sensitivity and specificity of ${ }^{18}$ F-fluorodeoxyglucose PET ( ${ }^{18}$ F-FDG-PET) for the detection of recurrent laryngeal carcinoma after radiotherapy was reported as 89 and $74 \%$, respectively, with a mean prevalence rate of recurrence of $50 \%$ [5].

Assessment of PET results is typically by visual interpretation, which is subject to interobserver variation. The extent to which results can be generalized, and thereby foresee the applicability of PET in daily clinical practice, depends on the degree of agreement among different observers. To investigate the interobserver agreement in reviewing $30{ }^{18} \mathrm{~F}$-FDG-PET scans of patients suspected of recurrent laryngeal carcinoma after radiotherapy, the results of 11 experienced nuclear medicine physicians from eight Dutch Head and Neck Society centers were analyzed. The interobserver variability showed a reasonable relation $(\kappa=0.55)$ between the observers in comparison to the reference standard (local recurrence within 6 months after PET) [20].

Furthermore, FDG-PET scan seems not only to be effective in selecting patients for direct laryngoscopy, but also less costly than initially performing a direct laryngoscopy under general anesthesia with biopsies in all patients with suspicion of recurrent laryngeal disease [21].

Recently, the multicenter randomized clinical RELAPS study (REcurrent LAryngeal carcinoma PET Study) was performed to determine whether ${ }^{18}$ F-FDG-PET can reliably select patients for direct laryngoscopy under general anesthesia when recurrent laryngeal carcinoma is 
suspected. Hundred and fifty patients were randomized to direct laryngoscopy under general anesthesia (conventional strategy), or to ${ }^{18}$ F-FDG-PET, only followed by laryngoscopy under general anesthesia in case of positive or equivocal findings on PET (PET-based strategy). If laryngoscopy did not reveal recurrent tumor, laryngoscopy was repeated within 6 weeks unless clinical signs and symptoms had diminished. In the PET-based strategy, patients with a negative PET were not subjected to additional investigations for at least 3 months, unless there was progression of symptoms. The achievable health gain comprises a reduction of the number of avoidable direct laryngoscopies and their consequences [22].

\section{Diffusion-weighted MRI (DW-MRI)}

DW-MRI is based on the relative mobility of water molecules in different tissues and provides information about the microenvironment such as cell density, cell integrity and vascularity [23]. It has only recently been introduced in the evaluation of HNSCC [24-26]. Several studies have already shown the benefit of DW-MRI in detecting residual or recurrent disease but included carcinomas of various anatomical head neck sites [25-27]. The sensitivity, specificity and accuracy in differentiating between residual or recurrent tumor and post-therapeutic changes was reported by Vandecaveye et al. [27] in 2012 as 95, 96 and $96 \%$, respectively. In a recently published prospective study [16], 46 patients with newly developed or worsening symptoms after (chemo)radiation of laryngeal $(n=30)$ and hypopharyngeal $(n=16)$ carcinomas underwent DWMRI to demonstrate or exclude residual or recurrent disease. The evaluation was performed by both qualitative (visual assessment) and quantitative analysis. The qualitative analysis allowed exclusion of tumors in all patients without residual or recurrent tumor and to detect residual or recurrent disease in 17 out of 18 patients with tumor; thus, the sensitivity, specificity and accuracy were 94, 100 and $98 \%$, respectively. The quantitative analysis [using the socalled apparent diffusion coefficient (ADC)] confirmed the ADC values to be lower in patients with than in those without residual or recurrent disease.

However, measurements of ADC values depend on the position, size and ease of delineation of the region of interest (ROI) that is placed within the lesion, and both intra- and interobserver reproducibility of ADC values can be problematic. Determining lesion borders may be complicated on DW-MRI, making it difficult to precisely localize the lesion and to obtain reliable ADC values. More accurate ADC values can be obtained by combining DWMRI with contrast-enhanced $\mathrm{T} 1$-weighted imaging to localize the lesion and to define ROIs. It is also unclear which DW-MRI technique (EPI or HASTE) is best suited to the head and neck area [28].

Although DW-MRI for the detection of recurrent laryngeal carcinoma after radiotherapy is promising, some technical problems, e.g., the difference in data obtained from different scanners and the optimal DW-technique, have to be solved before findings can be confirmed in prospective multicenter trials.

\section{Tumor spread/cr-T-classification}

At most centers, the classical surgical treatment of recurrent LSCC after (chemo)radiotherapy failure is salvage total laryngectomy. In those cases, the assessment of exact recurrent tumor extension is less important. A positive biopsy that proves tumor recurrence and CT or conventional MRI are sufficient in most cases to plan a salvage total laryngectomy. Currently, selected recurrences of early glottic cancer in particular are, when possible, treated by larynx-preserving salvage surgery [29]. In those cases, a precise evaluation of the recurrent tumor extension is mandatory for planning larynx-preserving salvage surgery. Nevertheless, the accuracy of recurrent tumor assessment and cr-T-classification by endoscopy and imaging studies has been debated only in a few articles and, to our knowledge, only two studies have been performed comparing endoscopic and radiologic findings with wholeorgan sections of salvage laryngectomy specimens [6, 30]. In one paper, a prospective study [30], the cr-T-classification was correct in 19 out of 32 cases $(59 \%)$ and in the other article, a retrospective study [31], in 21 out of 42 cases $(50 \%)$. In both series, most false classified recurrences were underestimated: 10 out of 13 and 18 out of 21 , respectively. The results in predicting tumor extent are disappointing. Small tumor foci, often localized in the subglottic region, and the extralaryngeal soft tissues as well as contralateral tumor spread are often undetectable by endoscopy and imaging studies. Therefore, to reduce the risk of local failures after larynx-preserving salvage surgery, intraoperative frozen-section analysis is mandatory for determining the surgical margins. Given these histopathological findings and the limitations of frozen-section analysis of surgical margins, the indication for larynxpreserving surgery in residual or recurrent LSCC after (chemo)radiotherapy must be considered carefully.

\section{Conclusions}

In case of suspicious symptoms or clinical findings after treatment of LSCC with (chemo)radiotherapy, the first aim of additional investigations must be to detect possible 
recurrent or residual disease as early as possible to increase the chance of successful salvage surgery. The second aim must be to avoid futile direct laryngoscopies under general anesthesia with biopsies, potentially exacerbating postradiotherapy changes. The accuracy of clinical symptoms, direct laryngoscopy with biopsy, CT and conventional MRI to detect recurrences is relatively low. FDG-PET-CT and DW-MRI studies seem more suitable to detect recurrences and to select patients with suspicion of recurrent or residual LSCC after (chemo)radiotherapy for direct laryngoscopy with biopsy. However, the literature is scanty. Most studies are retrospective and include HNSCC in general rather than LSCC only. Furthermore, the numbers of patients are limited in most studies. Unequivocal validated criteria for qualitative and quantitative interpretation of FDG-PET-CT and DW-MRI in detecting recurrences must be established [32]; thus, guaranteeing the reproducibility of results. Image fusion of PET combined with MRI might result in further improvement of the diagnostic accuracy. The combination of PET/MRI will hopefully decrease the number of falsepositive PET scans early after radiotherapy as a result of nonspecific tracer uptake caused by inflammation [33]. Moreover, FDG uptake can be correlated with the functional information obtained by new MR techniques.

More prospective, sufficiently powered studies on clearly defined patient cohorts are required to establish which are the optimal diagnostic procedures in the setting of suspicion on residual or recurrent disease after (chemo)radiation of LSCC.

\section{References}

1. Strojan P, Haigentz M Jr, Bradford CR et al (2013) Chemoradiotherapy vs. total laryngectomy for primary treatment of advanced laryngeal squamous cell carcinoma. Oral Oncol 49:283-286 (Editoral)

2. Forastiere AA, Goepfert H, Maor M et al (2003) Concurrent chemotherapy and radiotherapy for organ preservation in advanced laryngeal cancer. N Engl J Med 349:2091-2098

3. Forastiere AA, Zhang Q, Weber RS et al (2013) Long-term results of RTOG 91-11: a comparison of three nonsurgical treatment strategies to preserve the larynx in patients with locally advanced larynx cancer. J Clin Oncol 31:845-852

4. Genden EM, Ferlito A, Rinaldo A et al (2008) Recent changes in the treatment of patients with advanced laryngeal cancer. Head Neck 30:103-110

5. Brouwer J, Hooft L, Hoekstra OS et al (2008) Systematic review: accuracy of imaging tests in the diagnosis of recurrent laryngeal carcinoma after radiotherapy. Head Neck 30:889-897

6. Zbären P, Nuyens M, Curschmann J, Stauffer E (2007) Histologic characteristics and tumor spread of recurrent glottic carcinoma: analysis on whole-organ sections and comparison with tumor spread of primary glottic carcinoma. Head Neck 29:26-32

7. Brouwer J, Bodar EJ, de Bree R et al (2004) Detecting recurrent laryngeal carcinoma after radiotherapy: room for improvement. Eur Arch Otorhinolaryngol 261:417-422
8. Viani L, Stell PM, Dalby JE (1991) Recurrence after radiotherapy for glottic carcinoma. Cancer 67:577-584

9. Terhaard CH, Bongers V, van Rijk PP, Hordijk GJ (2001) F-18fluoro-deoxy-glucose positron-emission tomography scanning in detection of local recurrence after radiotherapy for laryngeal/ pharyngeal cancer. Head Neck 23:933-941

10. Crellin RP, Gaze MN, White A, Maran AG, MacDougall RH (1992) Salvage laryngectomy after radical radiotherapy for laryngeal carcinoma. Clin Otolaryngol Allied Sci 17:449-451

11. Ljumanovic R, Langendijk JA, Hoekstra OS, Knol DL, Leemans CR, Castelijns JA (2008) Pre- and post-radiotherapy MRI results as a predictive model for response in laryngeal carcinoma. Eur Radiol 18:2231-2240

12. Pameijer FA, Hermans R, Mancuso AA et al (1999) Pre- and post-radiotherapy computed tomography in laryngeal cancer: imaging-based prediction of local failure. Int J Radiat Oncol Biol Phys 45:359-366

13. Hermans R (2004) Post-treatment imaging of head and neck cancer. Cancer Imaging 4:6-15

14. de Bree R, van der Putten L, Brouwer J, Castelijns JA, Hoekstra OS, Leemans CR (2009) Detection of locoregional recurrent head and neck cancer after (chemo)radiotherapy using modern imaging. Oral Oncol 45:386-393

15. Briggs RJ, Gallimore AP, Phelps PD, Howard DJ (1993) Laryngeal imaging by computerized tomography and magnetic resonance following radiation therapy: a need for caution. J Laryngol Otol 107:565-568

16. Tshering Vogel DW, Zbaeren P, Geretschlaeger A, Vermathen $P$, De Keyzer F, Thoeny HC (2013) Diffusion-weighted MR imaging including bi-exponential fitting for the detection of recurrent or residual tumour after (chemo)radiotherapy for laryngeal and hypopharyngeal cancers. Eur Radiol 23:562-569

17. Fakhry N, Lussato D, Jacob T, Giorgi R, Giovanni A, Zanaret M (2007) Comparison between PET and PET/CT in recurrent head and neck cancer and clinical implications. Eur Arch Otorhinolaryngol 264:531-538

18. Halpern BS, Yeom K, Fueger BJ, Lufkin RB, Czernin J, AllenAuerbach M (2007) Evaluation of suspected local recurrence in head and neck cancer: a comparison between PET and PET/CT for biopsy proven lesions. Eur J Radiol 62:199-204

19. Gupta T, Master Z, Kannan S et al (2011) Diagnostic performance of post-treatment FDG PET or FDG PET/CT imaging in head and neck cancer: a systematic review and meta-analysis. Eur J Nucl Med Mol Imaging 38:2083-2095

20. van der Putten L, Hoekstra OS, de Bree R et al (2008) 2-Deoxy2[F-18]FDG-PET for detection of recurrent laryngeal carcinoma after radiotherapy: interobserver variability in reporting. Mol Imaging Biol 10:294-303

21. van Hooren AC, Brouwer J, de Bree R, Hoekstra OS, Leemans CR, Uyl-de Groot CA (2009) The cost-effectiveness of ${ }^{18}$ FDGPET in selecting patients with suspicion of recurrent laryngeal carcinoma after radiotherapy for direct laryngoscopy. Eur Arch Otorhinolaryngol 266:1441-1448

22. de Bree R, van der Putten L, Hoekstra OS et al (2007) A randomized trial of PET scanning to improve diagnostic yield of direct laryngoscopy in patients with suspicion of recurrent laryngeal carcinoma after radiotherapy. Contemp Clin Trials 28:705-712

23. Le Bihan D, Breton E, Lallemand D, Aubin ML, Vignaud J, Laval-Jeantet M (1988) Separation of diffusion and perfusion in intravoxel incoherent motion MR imaging. Radiology 168:497-505

24. Thoeny HC (2010) Diffusion-weighted MRI in head and neck radiology: applications in oncology. Cancer Imaging 10:209-214

25. Vandecaveye V, De Keyzer F, Nuyts S et al (2007) Detection of head and neck squamous cell carcinoma with diffusion weighted 
MRI after (chemo)radiotherapy: correlation between radiologic and histopathologic findings. Int $\mathrm{J}$ Radiat Oncol Biol Phys 67:960-971

26. Abdel Razek AA, Kandeel AY, Soliman N et al (2007) Role of diffusion-weighted echo-planar MR imaging in differentiation of residual or recurrent head and neck tumors and posttreatment changes. AJNR Am J Neuroradiol 28:1146-1152

27. Vandecaveye V, Dirix P, De Keyzer F et al (2012) Diffusionweighted magnetic resonance imaging early after chemoradiotherapy to monitor treatment response in head-and-neck squamous cell carcinoma. Int J Radiat Oncol Biol Phys 82:1098-1107

28. Verhappen MH, Pouwels PJ, Ljumanovic R et al (2012) Diffusion-weighted MR imaging in head and neck cancer: comparison between half-Fourier acquired single-shot turbo spin-echo and EPI techniques. AJNR Am J Neuroradiol 33:1239-1246

29. Agra IM, Ferlito A, Takes RP et al (2012) Diagnosis and treatment of recurrent laryngeal cancer following initial nonsurgical therapy. Head Neck 34:727-735
30. Han MW, Kim SA, Cho KJ et al (2013) Diagnostic accuracy of computed tomography findings for patients undergoing salvage total laryngectomy. Acta Otolaryngol Feb 28. [Epub ahead of print]

31. Zbären P, Christe A, Caversaccio MD, Stauffer E, Thoeny HC (2007) Pre-therapeutic staging of recurrent laryngeal carcinoma: clinical findings and imaging studies compared with histopathology. Otolaryngol Head Neck Surg 137:487-491

32. de Bree R, Hoekstra OS (2010) Predictive value of fluorodeoxyglucose uptake in head and neck cancer: importance of standardization: editorial on EAORL-D-10-00277. Eur Arch Otorhinolaryngol 267:1491-1493

33. Srinivasan A, Mohan S, Mukherji SK (2012) Biologic imaging of head and neck cancer: the present and the future. AJNR Am J Neuroradiol 33:586-594 